\title{
The Catalonian Crises through Google Searches: A Regional Perspective
}

\author{
Artola, Concha and Pérez, Javier J.
}

DG Econonomics and Statistics, Banco de España

\begin{abstract}
In this paper we focus in the period of political turmoil starting in September 2017 in Catalonia. Our research question is the following: can the Catalan crisis be tracked by the searches done by the public on different consumption items in the Internet? We do so by focusing in two set of consumption categories: Travel to Catalonia from the main international markets (France, Germany and United Kingdom) and searches on the main consumption categories done from Catalonia and from other five big regions (Madrid, Valencia, Aragón, Andalucía and Basque Country). The preliminary results show that the uncertainty in the political situation has translated unto a decline in searches on terms associated with tourism activities in Barcelona, one broad measure shows that searches for the term "Barcelona hotel" has declined by 12\%, year on year for September 2017 to January 2018, by comparison searches for hotel in other comparable Spanish regions have increased slightly. When comparing searches done from Catalonia with other regions through simple time series models, a sizeable negative residual for Catalonia is present in October 2017 -the most difficult period in the Catalan conundrum- which is not observed in other geographical areas. This is the case for some search topics associated to durable goods and Catering and Accommodation services. The political turmoil in Catalonia had significant negative effects in two consumption categories: Theaters and Restaurants.
\end{abstract}

Keywords: Google Trends, International Tourism, Catalonia, Private Consumption 